\title{
TEMPERED REPRESENTATIONS AND NILPOTENT ORBITS
}

\author{
BENJAMIN HARRIS
}

\begin{abstract}
Given a nilpotent orbit $\mathcal{O}$ of a real, reductive algebraic group, a necessary condition is given for the existence of a tempered representation $\pi$ such that $\mathcal{O}$ occurs in the wave front cycle of $\pi$. The coefficients of the wave front cycle of a tempered representation are expressed in terms of volumes of precompact submanifolds of an affine space.
\end{abstract}

\section{INTRODUCTION}

Let $G$ be a real, reductive algebraic group with Lie algebra $\mathfrak{g}$, and let $\pi$ be an irreducible, admissible representation of $G$. The wave front cycle of $\pi$, denoted $\mathrm{WF}(\pi)$, is an integral linear combination of nilpotent coadjoint orbits defined in [2], 13 .

Theorem 1.1. Suppose $\mathcal{O}$ is an orbit contained in $W F(\pi)$ for a tempered representation $\pi$, let $\nu \in \mathcal{O}$, and let $L$ be a Levi factor of $Z_{G}(\nu)$. Then $L / Z(G)$ is compact.

This theorem was conjectured by David Vogan. It makes precise the widely held intuition that orbits occurring in the wave front cycles of tempered representations are necessarily large.

In [1] Bala and Carter attempted a uniform understanding of nilpotent orbits in complex semisimple Lie algebras. They observed that for every nilpotent orbit $\mathcal{O}$, there is a unique (up to conjugacy) maximal complex Levi subalgebra $\mathfrak{l}$ meeting $\mathcal{O}$. Moreover, $\mathcal{O} \cap \mathfrak{l}$ is a single orbit for the adjoint group of $\mathfrak{l}$. Bala and Carter defined a nilpotent orbit to be distinguished if it does not meet a proper Levi subalgebra, and they observed that to parametrize nilpotent orbits it is enough to parametrize distinguished nilpotent orbits in every Levi subalgebra.

Motivated by the work of Bala and Carter, Noël defined a nilpotent orbit for a real, reductive algebraic group to be noticed if it does not meet a proper Levi subalgebra [8]. By Levi subalgebra, we mean the Levi factor of a real parabolic subalgebra.

If $\mathcal{O}_{\nu}$ is a nilpotent orbit, then it is not hard to show that $Z_{G}(\nu)$ is compact modulo $Z(G)$ iff $\mathcal{O}_{\nu}$ is a noticed nilpotent orbit. Thus, we have an alternate way of stating Theorem 1.1.

Received by the editors October 19, 2010 and in revised form, May 28, 2011 and September 18, 2011

2010 Mathematics Subject Classification. Primary 22E46; Secondary 43A65, 22E45.

Key words and phrases. Tempered representation, discrete series representation, wave front cycle, associated variety, reductive lie group, real reductive algebraic group, nilpotent orbit, distinguished nilpotent orbit, noticed nilpotent orbit, coadjoint orbit.

(C)2012 American Mathematical Society Reverts to public domain 28 years from publication 
Corollary 1.2. If $\mathcal{O} \subset \mathfrak{g}^{*}$ is a nilpotent coadjoint orbit occurring in the wave front cycle of a tempered representation, then $\mathcal{O}$ does not meet $\mathfrak{m}^{*}$ for any proper Levi subalgebra $\mathfrak{m} \subset \mathfrak{g}$.

Note that this corollary relates a set of nilpotent orbits that arises naturally in representation theory with a set of nilpotent orbits that arises naturally in structure theory.

For $p$-adic groups, similar results were obtained much earlier. In [6], Moeglin shows that the $p$-adic analogue for supercuspidal representations follows from results of [7. Similarly, Moeglin proves the result for tempered representations of classical $p$-adic groups in [6].

The purpose of this paper is to show that there is a simple proof of Theorem 1.1, which is in the spirit of the Kostant-Kirillov orbit method.

In [10] and [1], Rossmann associated to each irreducible, tempered representation $\pi$ a finite union of regular coadjoint orbits we call $\mathcal{O}_{\pi}$. If $\pi$ has regular infinitesimal character, then $\mathcal{O}_{\pi}$ is a single coadjoint orbit. For each nilpotent coadjoint orbit, $\mathcal{O} \subset \mathfrak{g}^{*}$, fix $X \in \mathcal{O}$. Identify $\mathfrak{g} \cong \mathfrak{g}^{*}$ via a $G$-equivariant isomorphism, and let $\{X, H, Y\}$ be an $\mathfrak{s l}_{2}$-triple containing $X$. Put $S_{X}=X+Z_{\mathfrak{g}}(Y)$.

Theorem 1.3. There exists a canonical measure on $S_{X} \cap \mathcal{O}_{\pi}$ such that

$$
W F(\pi)=\sum_{\mathcal{O}_{\pi} \cap S_{X}} \operatorname{vol}\left(\mathcal{O}_{\pi} \cap S_{X}\right) \mathcal{O}_{X} .
$$

The sum is over nilpotent coadjoint orbits $\mathcal{O}_{X}$ such that $\mathcal{O}_{\pi} \cap S_{X}$ is precompact.

If $\pi$ has regular infinitesimal character, then "precompact" may be replaced by "compact" in the above theorem. In the case where $G$ is compact, the wave front cycle of $\pi$ is $\operatorname{dim}(\pi) \cdot 0$ where 0 denotes the zero orbit. In this case, our formula reduces to the well-known observation of Kirillov that the symplectic volume of the coadjoint orbit associated to $\pi$ is the dimension of $\pi$ (see, for instance, page 173 of [5]).

The reader will see in section four that the compactness in Theorem 1.1 is a consequence of the compactness of the manifold $\mathcal{O}_{\pi} \cap S_{X}$ in the case of regular infinitesimal character.

All of these results should be true for an arbitrary reductive Lie group. We only state them in the case of real, reductive algebraic groups because we use results of [10, 11, and [12, which are only stated for real, reductive algebraic groups. However, the author believes that these results should be true for an arbitrary reductive Lie group.

\section{RaO's Lemma and three Corollaries of Barbasch and Vogan}

Let $\mathcal{O} \subset \mathfrak{g}^{*}$ be a coadjoint orbit. The Kostant-Kirillov symplectic form $\omega$ is defined on $\mathcal{O}$ by the formula

$$
\omega_{\lambda}\left(\operatorname{ad}_{X}^{*} \lambda, \operatorname{ad}_{Y}^{*} \lambda\right)=\lambda([X, Y]) .
$$

The top dimensional form

$$
\frac{\omega^{m}}{m !(2 \pi)^{m}}
$$

on $\mathcal{O}$ gives rise to the canonical measure on $\mathcal{O}$. Here $m=\frac{\operatorname{dim} \mathcal{O}}{2}$. We will often abuse notation and write $\mathcal{O}$ for the orbit as well as the canonical measure on the orbit. 
In this section, we recall an unpublished lemma of Rao and three corollaries of Barbasch and Vogan. All of this material can be found on pages 46, 47, and 48 of [2]. However, unlike the previous treatment, we need to carefully keep track of certain constants for our applications. Thus, we provide updated statements, and for the convenience of the reader we provide sketches of updated proofs.

Identify $\mathfrak{g} \cong \mathfrak{g}^{*}$ via a $G$-equivariant isomorphism. Let $\mathcal{O}_{X}$ be a nilpotent orbit in $\mathfrak{g}^{*} \cong \mathfrak{g}$, and let $\{X, H, Y\}$ be an $\mathfrak{s l}_{2}$-triple with nilpositive element $X$. Put $S_{X}=X+Z_{\mathfrak{g}}(Y)$. The map

$$
\phi: G \times S_{X} \rightarrow \mathfrak{g}^{*}
$$

given by $\phi:(g, \xi) \mapsto g \cdot \xi$ is a submersion. In particular, every orbit $\mathcal{O}_{\nu} \subset G \cdot S_{X}$ is transverse to $S_{X}$, and $G \cdot S_{X} \subset \mathfrak{g}^{*}$ is open.

Fix a Haar measure on $G$. This choice determines a Lebesgue measure on $\mathfrak{g} \cong \mathfrak{g}^{*}$. If $\xi \in S_{X}$, then we have a direct sum decomposition

$$
\mathfrak{g}=[\mathfrak{g}, X] \oplus Z_{\mathfrak{g}}(Y) \cong T_{X} \mathcal{O}_{X} \oplus T_{\xi} S_{X}
$$

We then obtain a Lebesgue measure on $S_{X}$ as the "quotient" of the Lebesgue measure on $\mathfrak{g}$ and the canonical measure on $\mathcal{O}_{X} \subset \mathfrak{g}^{*}$. Further, given $\nu \in \mathfrak{g}^{*}$, denote by $\mathcal{F}_{\nu}$ the fiber over $\nu$ under the map $\phi$. If $g \cdot \xi=\nu$, then we have an exact sequence

$$
0 \rightarrow T_{\nu}^{*}\left(G \cdot S_{X}\right) \rightarrow T_{(g, \xi)}^{*}\left(G \times S_{X}\right) \rightarrow T_{(g, \xi)}^{*} \mathcal{F}_{\nu} \rightarrow 0
$$

This exact sequence together with the above remarks and our choice of Haar measure on $G$ determine a smooth measure on $\mathcal{F}_{\nu}$. Moreover, integration against these measures on the fibers of $\phi$ yields a continuous surjective map

$$
\phi_{*}: C_{c}^{\infty}\left(G \times S_{X}\right) \longrightarrow C_{c}^{\infty}\left(G \cdot S_{X}\right) .
$$

Dualizing, we get an injective pullback map on distributions

$$
\phi^{*}: D\left(G \cdot S_{X}\right) \rightarrow D\left(G \times S_{X}\right) .
$$

Now, we are ready to state Rao's lemma.

Lemma 2.1 (Rao). If $\nu \in S_{X}$, then there exists a smooth measure $m_{\nu, X}$ on $\mathcal{O}_{\nu} \cap S_{X}$ such that

$$
\phi^{*}\left(\mathcal{O}_{\nu}\right)=m_{G} \otimes m_{\nu, X} .
$$

Here $m_{G}$ denotes the fixed choice of Haar measure on $G$. Although $\phi^{*}$ depends on this choice of Haar measure, $m_{\nu, X}$ does not.

One can write down $m_{\nu, X}$ by giving a top dimensional form on $\mathcal{O}_{\nu} \cap S_{X}$, welldefined up to sign. Essentially, we just divide the canonical measure on $\mathcal{O}_{\nu}$ by the canonical measure on $\mathcal{O}_{X}$. More precisely, the composition of the inclusion $[\mathfrak{g}, \nu] \hookrightarrow \mathfrak{g}$ and the projection defined by the decomposition $\mathfrak{g}=[\mathfrak{g}, X] \oplus Z_{\mathfrak{g}}(Y)$ yields a map

$$
T_{\nu} \mathcal{O}_{\nu} \cong[\mathfrak{g}, \nu] \rightarrow[\mathfrak{g}, X] \cong T_{X} \mathcal{O}_{X}
$$

This map is a surjection because $\mathcal{O}_{\nu}$ is transverse to $S_{X}$. It pulls back to an exact sequence

$$
0 \rightarrow T_{X}^{*} \mathcal{O}_{X} \rightarrow T_{\nu}^{*} \mathcal{O}_{\nu} \rightarrow T_{\nu}^{*}\left(\mathcal{O}_{\nu} \cap S_{X}\right) \rightarrow 0 .
$$

The canonical measures on $\mathcal{O}_{\nu}$ and $\mathcal{O}_{X}$ determine top dimensional alternating tensors up to sign on $T_{\nu}^{*} \mathcal{O}_{\nu}$ and $T_{X}^{*} \mathcal{O}_{X}$. Hence, our exact sequence gives a top dimensional, alternating tensor on $T_{\nu}^{*}\left(\mathcal{O}_{\nu} \cap S_{X}\right)$, well-defined up to sign. 
We will need three corollaries of Barbasch and Vogan. Let $\mathcal{N} \subset \mathfrak{g}^{*}$ be the nilpotent cone. If $\nu \in \mathfrak{g}^{*}$, define

$$
\mathcal{N}_{\nu}=\mathcal{N} \cap \overline{\bigcup_{t>0} \mathcal{O}_{t \nu}}
$$

Corollary 2.2 (Barbasch and Vogan). We have four statements.

(a) If $\mathcal{O}_{X}$ is a nilpotent orbit, then $\mathcal{O}_{X} \subset \mathcal{N}_{\nu}$ if, and only if $\mathcal{O}_{\nu} \cap S_{X} \neq \emptyset$.

(b) An orbit $\mathcal{O}_{X} \subset \mathcal{N}_{\nu}$ is open if, and only if $\mathcal{O}_{\nu} \cap S_{X}$ is precompact.

(c) If $\nu$ is semisimple, then $\mathcal{O}_{X} \subset \mathcal{N}_{\nu}$ is open if, and only if $\mathcal{O}_{\nu} \cap S_{X}$ is compact.

(d) Further, $\mathcal{O}_{X} \cap S_{X}=\{X\}$ for any nilpotent orbit $\mathcal{O}_{X}$.

We sketch a proof. Note $G \cdot S_{X} \subset \mathfrak{g}^{*}$ is an open subset containing $\mathcal{O}_{X}$; thus, $\mathcal{O}_{X} \subset$ $\mathcal{N}_{\nu}$ iff $\mathcal{O}_{t \nu} \cap S_{X} \neq \emptyset$ for sufficiently small $t>0$. However, if $\gamma_{t}=\exp \left(-\frac{1}{2}(\log (t)) H\right)$, then

$$
\mathcal{O}_{t \nu} \cap S_{X}=X+t \gamma_{t}\left(\mathcal{O}_{\nu} \cap S_{X}-X\right) .
$$

In particular, $\mathcal{O}_{\nu} \cap S_{X} \neq \emptyset$ iff $\mathcal{O}_{t \nu} \cap S_{X} \neq \emptyset$ for any $t>0$. This verifies part (a).

For parts (b) and (c), one shows that $\mathcal{O}_{\nu} \cap S_{X}$ bounded implies that $S_{X} \cap$ $\mathcal{N}_{\nu}=\{X\}$ and $\mathcal{O}_{\nu} \cap S_{X}$ unbounded implies that $S_{X} \cap \mathcal{N}_{\nu}$ is unbounded. This follows from a straightforward calculation utilizing the $\operatorname{ad}_{H}$-decomposition of $Z_{\mathfrak{g}}(Y)$ into eigenspaces with nonpositive eigenvalues and the above relationship between $\mathcal{O}_{\nu} \cap S_{X}, \mathcal{O}_{t \nu} \cap S_{X}$, and $\gamma_{t}$. Using that $\left(G \cdot S_{X}\right) \cap \mathcal{N}$ is the union of nilpotent orbits containing $\mathcal{O}_{X}$ in their closures, parts (b) and (c) follow.

If we let $\nu=X$ in the last paragraph, we arrive at part (d).

Corollary 2.3 (Barbasch and Vogan). Let $n=\frac{1}{2}\left(\operatorname{dim} \mathcal{O}_{\nu}-\operatorname{dim} \mathcal{N}_{\nu}\right)$. Then

$$
\lim _{t \rightarrow 0^{+}} t^{-n} \mathcal{O}_{t \nu}=\sum_{\substack{\mathcal{O}_{X} \subset \mathcal{N}_{\nu} \\ \operatorname{dim} \mathcal{O}_{X}=\operatorname{dim} \mathcal{N}_{\nu}}} \operatorname{vol}\left(\mathcal{O}_{\nu} \cap S_{X}\right) \mathcal{O}_{X}
$$

The volumes are computed with respect to the measures defined in Lemma 2.1. Moreover, the Fourier transform of the right-hand side is the first nonzero term in the asymptotic expansion of the generalized function $\widehat{\mathcal{O}_{\nu}}$.

Again, we sketch a proof. Fix $X$, a nilpotent element with $\mathcal{O}_{X} \subset \mathcal{N}_{\nu}$ open, and let $m=\frac{1}{2}\left(\operatorname{dim} \mathcal{O}_{\nu}-\operatorname{dim} \mathcal{O}_{X}\right)$. We first show

$$
\lim _{t \rightarrow 0^{+}} t^{-m} \mathcal{O}_{t \nu}=\operatorname{vol}\left(\mathcal{O}_{\nu} \cap S_{X}\right) \mathcal{O}_{X}
$$

on the open set $G \cdot S_{X}$. By Rao's lemma, $\phi^{*}\left(\mathcal{O}_{\nu}\right)=m_{G} \otimes m_{\nu, X}$. Thus, it is enough to show

$$
\lim _{t \rightarrow 0^{+}} t^{-m} m_{G} \otimes m_{t \nu, X}=\operatorname{vol}\left(\mathcal{O}_{\nu} \cap S_{X}\right) m_{G} \otimes \delta_{X}
$$

if $\mathcal{O}_{X} \subset \mathcal{N}_{\nu}$ is an open orbit. Note that the support of the measure $m_{t \nu, X}$ is the precompact set $\mathcal{O}_{t \nu} \cap S_{X}$, and the precompact sets $\mathcal{O}_{t \nu} \cap S_{X}$ converge uniformly to $\delta_{X}$ by the above relationship between $\mathcal{O}_{\nu} \cap S_{X}, \mathcal{O}_{t \nu} \cap S_{X}$, and $\gamma_{t}$. Thus, it is enough to show

$$
t^{-m} \operatorname{vol}\left(\mathcal{O}_{t \nu} \cap S_{X}\right)=\operatorname{vol}\left(\mathcal{O}_{\nu} \cap S_{X}\right) .
$$

This follows from a straightforward computation utilizing the above definition of the measures $m_{t \nu, X}$.

By Theorem 3.2 of [2], the distribution $\widehat{\mathcal{O}_{\nu}}$ has an asymptotic expansion at the origin

$$
t^{r} \widehat{\mathcal{O}_{t \nu}} \sim t^{l} D_{l}+\cdots
$$


where $D_{l}$ is the leading term and $r$ is the number of positive roots of $G$. If we show $n=l-r$, then our limit will exist everywhere. If $n>l-r$, then the limit $\lim _{t \rightarrow 0^{+}} t^{n} \widehat{\mathcal{O}_{t \nu}}$ must be zero everywhere. However, we have seen that the Fourier transform of this limit is nonzero on $\mathcal{O}_{X}$ whenever $\mathcal{O}_{X} \subset \mathcal{N}_{\nu}$ is of maximal dimension.

The limit $\lim _{t \rightarrow 0^{+}} t^{-l+r} \mathcal{O}_{t \nu}$ must exist and be nonzero. However, if $n<l-r$, then the homogeneity degree of this invariant distribution and Corollary 3.9 of 2 ] imply that such a distribution would have to be supported on orbits of dimension greater than $\operatorname{dim} \mathcal{N}_{\nu}$. But, this is impossible since the $\operatorname{limit}_{t \rightarrow 0^{+}} t^{-l+r} \mathcal{O}_{t \nu}$, if it exists, is clearly supported in $\mathcal{N}_{\nu}$. Hence, $n=l-r$ and the limit $\lim _{t \rightarrow 0^{+}} t^{-n} \mathcal{O}_{t \nu}$ exists.

Now, let $k=\operatorname{dim} \mathcal{N}_{\nu}$, and let $\mathcal{N}_{k}$ be the union of nilpotent orbits of dimension at least $k$. We have shown that our desired limit formula holds on $\mathcal{N}_{k}$. However, in theory the limit could differ from

$$
\sum_{\substack{\mathcal{O}_{X} \subset \mathcal{N}_{\nu} \mathcal{N}_{\nu} \\ \operatorname{dim} \mathcal{O}_{X}=\operatorname{dim} \mathcal{N}_{\nu}}} \operatorname{vol}\left(\mathcal{O}_{\nu} \cap S_{X}\right) \mathcal{O}_{X}
$$

by a distribution $u$ supported on orbits of dimension less than $\mathcal{N}_{\nu}$. However, we deduce $u=0$ from Corollary 3.9 of 2 after checking the homogeneity degree of the terms in our limit formula.

Corollary 2.4 (Barbasch and Vogan). Suppose $\mathfrak{h} \subset \mathfrak{g}$ is a Cartan, and let $C \subset$ $\left(\mathfrak{h}^{*}\right)^{\prime}$ be a connected component of the regular set. If $\nu, \lambda \in C$, then $\mathcal{N}_{\nu}=\mathcal{N}_{\lambda}$.

Suppose $\mathcal{O}_{X} \subset \mathcal{N}_{\nu}$ is an open orbit, let $n=\frac{1}{2}\left(\operatorname{dim} \mathcal{O}_{\nu}-\operatorname{dim} \mathcal{O}_{X}\right)$, and observe $\lim _{t \rightarrow 0^{+}} t^{-n} \mathcal{O}_{t \nu}=\left.\lim _{t \rightarrow 0^{+}} \frac{1}{n !} \partial(\nu)^{n}\right|_{t \nu} \mathcal{O}_{t \nu}$ on $G \cdot S_{X}$ by the proof of Corollary 2.3. By Lemma 22 of [4],

$$
\left.\lim _{t \rightarrow 0^{+}} \partial(\nu)^{n}\right|_{t \nu} \mathcal{O}_{t \nu}=\left.\lim _{t \rightarrow 0^{+}} \partial(\nu)^{n}\right|_{t \lambda} \mathcal{O}_{t \lambda}
$$

on $G \cdot S_{X}$ if $\nu, \lambda \in C$. Clearly the support of $\left.\lim _{t \rightarrow 0^{+}} \partial(\nu)^{n}\right|_{t \lambda} \mathcal{O}_{t \lambda}$ must be contained in $\mathcal{N}_{\lambda}$. Thus, the explicit formula for the limit on the left on $G \cdot S_{X}$ for open orbits $\mathcal{O}_{X} \subset \mathcal{N}_{\nu}$ in the proof of Corollary 2.3 then implies $\mathcal{O}_{X} \subset \mathcal{N}_{\lambda}$ whenever $\mathcal{O}_{X} \subset \mathcal{N}_{\nu}$ is open. Thus, we deduce $\mathcal{N}_{\nu} \subset \mathcal{N}_{\lambda}$. By symmetry we have equality.

\section{ROSSMANN'S CHARACTER FORMULA}

Let $V$ be a finite dimensional, real vector space, and let $\mu$ be a smooth, rapidly decreasing measure on $V$. Then the Fourier transform of $\mu$ is defined to be

$$
\widehat{\mu}(l)=\int_{V} e^{i\langle l, X\rangle} d \mu(X) .
$$

Note $\widehat{\mu}$ is a Schwartz function on $V^{*}$. Given a tempered distribution $D$ on $V^{*}$, its Fourier transform $\widehat{D}$ is a tempered, generalized function on $V$ defined by

$$
\langle\widehat{D}, \mu\rangle:=\langle D, \widehat{\mu}\rangle .
$$

Let $G$ be a real, reductive algebraic group, and let $\pi$ be an irreducible, tempered representation of $G$ with character $\Theta_{\pi}$. Let $\theta_{\pi}$ be the Lie algebra analogue of the character of $\pi$. Rossmann associated to $\pi$ a finite union of regular, coadjoint orbits $\mathcal{O}_{\pi} \subset \mathfrak{g}^{*}$. He proved the following theorem [10, 11]. 
Theorem 3.1 (Rossmann). As generalized functions, we have

$$
\widehat{\mathcal{O}_{\pi}}=\theta_{\pi} \text {. }
$$

Here $\widehat{\mathcal{O}_{\pi}}$ denotes the Fourier transform of the canonical measure on $\mathcal{O}_{\pi}$.

If $\mathcal{O}_{\pi}=\mathcal{O}_{\nu}$ is a single orbit, then the leading term of the asymptotic expansion of $\widehat{\mathcal{O}_{\pi}}=\widehat{\mathcal{O}_{\nu}}$ is

$$
\sum_{\substack{\mathcal{O}_{X} \subset \mathcal{N}_{\nu} \\ \operatorname{dim} \mathcal{O}_{X}=\operatorname{dim} \mathcal{N}_{\nu}}} \operatorname{vol}\left(\mathcal{O}_{\pi} \cap S_{X}\right) \widehat{\mathcal{O}_{X}}
$$

by Corollary 2.3. More generally, suppose $\mathcal{O}_{\pi}=\bigcup \mathcal{O}_{\nu_{i}}$, and define $\operatorname{AS}(\pi)=\bigcup \mathcal{N}_{\nu_{i}}$. Then the leading term of $\widehat{\mathcal{O}_{\pi}}$ is a sum over the leading terms of the $\widehat{\mathcal{O}_{\nu_{i}}}$ of minimal degree. This is just the sum over $\operatorname{vol}\left(\mathcal{O}_{\pi} \cap S_{X}\right) \widehat{\mathcal{O}_{X}}$ where $\mathcal{O}_{X} \subset \operatorname{AS}(\pi)$ varies over orbits of maximal dimension. Hence, we have shown

$$
\mathrm{WF}(\pi)=\sum_{\substack{\mathcal{O}_{X} \subset \operatorname{AS}(\pi) \\ \operatorname{dim} \mathcal{O}_{X}=\operatorname{dim~AS}(\pi)}} \operatorname{vol}\left(\mathcal{O}_{\pi} \cap S_{X}\right) \mathcal{O}_{X} .
$$

Thus, to prove Theorem 1.3, we need only show that $\mathcal{O}_{X} \subset \operatorname{AS}(\pi)$ is of maximal dimension iff $\mathcal{O}_{\pi} \cap S_{X}$ is precompact and nonempty. If $\mathcal{O}_{X} \subset \operatorname{AS}(\pi)$ is of maximal dimension, then $\mathcal{O}_{X} \cap \mathcal{N}_{\nu_{i}}$ is open for every $i$ and nonempty for at least one $i$, and by Corollary 2.2, $\mathcal{O}_{\nu_{i}} \cap S_{X}$ is precompact for every $i$ and nonempty for at least one $i$. We conclude $\mathcal{O}_{\pi} \cap S_{X}$ is precompact and nonempty.

Conversely, suppose $\mathcal{O}_{\pi} \cap S_{X}$ is precompact and nonempty. Then $\mathcal{O}_{\nu_{i}} \cap S_{X}$ is precompact for every $i$ and nonempty for some $i$. Corollary 2.2 tells us that $\mathcal{O}_{X} \subset \mathcal{N}_{\nu_{i}}$ is open for some $i$ and $\mathcal{O}_{X} \cap \mathcal{N}_{\nu_{i}}$ is open for every $i$. Hence, we have that $\mathcal{O}_{X} \subset \operatorname{AS}(\pi)$ is open. But, by Theorem $D$ of $[12, \operatorname{AS}(\pi)$ is the closure of the union of the orbits in $\operatorname{AS}(\pi)$ of maximal dimension. Thus, $\mathcal{O}_{X} \subset \operatorname{AS}(\pi)$ open implies that $\mathcal{O}_{X} \subset \mathrm{AS}(\pi)$ is of maximal dimension.

\section{Proof of Theorem 1.1}

In this section we prove Theorem 1.1. It is enough to prove the theorem when $\pi$ is an irreducible, tempered representation. Let $\mathcal{O}_{X} \subset \operatorname{AS}(\pi)$ be an open orbit, and identify $\mathfrak{g} \cong \mathfrak{g}^{*}$ via a $G$-equivariant isomorphism. Let $L \subset Z_{G}(X)$ be a Levi factor. Then there exists an $\mathfrak{s l}_{2}$-triple $\{X, H, Y\}$ such that $L=Z_{G}\{X, H, Y\}$. To prove the theorem, we must show

$$
Z_{G}\{X, H, Y\} / Z(G) \text { is compact. }
$$

Now, if $\mathcal{O}_{\pi}=\bigcup \mathcal{O}_{\nu_{i}}$, then $\operatorname{AS}(\pi)=\bigcup \mathcal{N}_{\nu_{i}}$. Any open orbit in $\operatorname{AS}(\pi)$ must be open in some $\mathcal{N}_{\nu_{i}}$. Hence, it is enough to prove (*) whenever $\mathcal{O}_{X}$ is open in $\mathcal{N}_{\nu}$ for a regular element $\nu \in \mathfrak{g}^{*}$.

Next, supplement A and supplement $\mathrm{C}$ of [9] imply that every regular orbit $\mathcal{O}_{\nu}$ can be written as a limit of regular semisimple orbits in the following sense. Let $\xi \in \mathfrak{g}^{*} \cong \mathfrak{g}$ be a semisimple element in the closure of $\mathcal{O}_{\nu}$, and let $\mathfrak{h} \subset \mathfrak{g}$ be a fundamental Cartan in $Z_{\mathfrak{g}}(\xi)$. Then there exists a connected component $C \subset\left(\mathfrak{h}^{*}\right)^{\prime}$ such that

$$
\lim _{\substack{\lambda \in C \\ \lambda \rightarrow \xi}} \mathcal{O}_{\lambda}=\mathcal{O}_{\nu}
$$

Hence, $\mathcal{N}_{\nu}=\mathcal{N}_{\lambda}$ for some regular semisimple element $\lambda$, and it is enough to prove $(*)$ for $\mathcal{O}_{X}$ open in $\mathcal{N}_{\lambda}$ with $\lambda$ regular, semisimple. 
Fix $X$ and suppose $\mathcal{O}_{X} \subset \mathcal{N}_{\lambda}$ is open for some $\lambda$ regular semisimple. By Corollary 2.2, $\mathcal{O}_{\lambda} \cap S_{X}$ is compact and nonempty. Now, $L=Z_{G}\{X, H, Y\}$ acts on this space, and $L$ must have at least one closed orbit (for instance, one can take an orbit of minimal dimension). Without loss of generality, we make it $L \cdot \lambda \subset \mathcal{O}_{\lambda} \cap S_{X}$. Choose a Cartan $\mathfrak{h}$ and a component of the regular set $C \subset\left(\mathfrak{h}^{*}\right)^{\prime}$ such that $\lambda \in C$. If $\xi \in U_{1}=G \cdot C$, then $\mathcal{O}_{X} \subset \mathcal{N}_{\lambda}=\mathcal{N}_{\xi}$ is open by Corollary 2.4. It then follows from Corollary 2.2 that $\mathcal{O}_{\xi} \cap S_{X}$ is compact for all $\xi$ in the open set $U_{1}$. Define $U=U_{1} \cap S_{X}$, an open subset of $S_{X}$.

Now, $L / Z_{G}(\lambda) \cong L \cdot \lambda \subset \mathcal{O}_{\lambda} \cap S_{X}$ is a closed subset of a compact set; hence, $L / Z_{G}(\lambda)$ is compact. Note that $Z_{G}(\lambda) \subset G$ is a Cartan since $\lambda$ is regular, semisimple. Thus, $Z_{L}(\lambda) \subset Z_{G}(\lambda)$ is abelian and consists of semisimple elements. Hence, the connected component of the identity $Z_{L}(\lambda)_{0}$ must be contained in a Cartan $B$ of $L$. We have seen that $L / Z_{L}(\lambda)$ is compact; hence, $L / Z_{L}(\lambda)_{0}$ is compact because $Z_{L}(\lambda)$ has finitely many components. This implies $L / B$ is compact and finally

$$
L / Z(L)
$$

is compact since semisimple groups are compact iff they are compact modulo a Cartan.

Because the fibers of the projection

$$
L / Z(G) \rightarrow L / Z(L)
$$

are homeomorphic to $Z(L) / Z(G)$, to show that $L / Z(G)$ is compact, it is enough to show $Z(L) / Z(G)$ is compact.

The following lemma is the key step in proving that $Z(L) / Z(G)$ is compact.

Lemma 4.1. Let $Z(\mathfrak{l})$ denote the center of $\mathfrak{l}=\operatorname{Lie}(L)$, and let $Z(\mathfrak{g})$ denote the center of $\mathfrak{g}$. Then

$$
\bigcap_{\xi \in U}\left(Z(\mathfrak{l}) \cap Z_{\mathfrak{l}}(\xi)\right)=Z(\mathfrak{g}) .
$$

Proof. Clearly the right-hand side is contained in the left-hand side. To show the other direction, suppose $W \in\left(Z(\mathfrak{l}) \cap Z_{\mathfrak{l}}(\xi)\right)$ for all $\xi \in U$. We will show $W \in Z(\mathfrak{g})$. Since $W \in \mathfrak{l}$, we know $X, H, Y \in Z_{\mathfrak{g}}(W)$. Further, $Z_{\mathfrak{g}}(W) \cap Z_{\mathfrak{g}}(Y) \subset Z_{\mathfrak{g}}(Y)$ is a vector subspace containing $U-X$ since $W \in Z_{\mathfrak{l}}(\xi)$ for $\xi \in U$ and $X \in Z_{\mathfrak{g}}(W)$. Since $U-X \subset Z_{\mathfrak{g}}(Y)$ is an open subset, we must have

$$
Z_{\mathfrak{g}}(W) \supset Z_{\mathfrak{g}}(Y) .
$$

Now, view $\mathfrak{g}$ as a finite dimensional module for $\operatorname{Span}_{\mathbb{R}}\{X, H, Y\} \cong \mathfrak{s l}_{2} \mathbb{R}$. Note $Z_{\mathfrak{g}}(W) \subset \mathfrak{g}$ is a subalgebra and a submodule for $\operatorname{Span}_{\mathbb{R}}\{X, H, Y\}$ since $X, H, Y \in$ $Z_{\mathfrak{g}}(W)$. But, the lowest weight vectors of each irreducible summand of $\mathfrak{g}$ are in $Z_{\mathfrak{g}}(W)$ since $Z_{\mathfrak{g}}(W) \supset Z_{\mathfrak{g}}(Y)$, and the lowest weight vectors of any finite dimensional $\mathfrak{s l}_{2}$ module generate the entire module. Thus, $Z_{\mathfrak{g}}(W)=\mathfrak{g}$ and $W \in Z(\mathfrak{g})$ as desired.

Before we get back to showing that $Z(L) / Z(G)$ is compact, we need two general remarks. First, suppose $A$ is an abelian, real algebraic group, suppose $\phi: A \rightarrow$ $\operatorname{Aut}(V)$ is a representation of $A$ on a real vector space $V$, and suppose $S \subset V$ is a compact $A$-stable subset of $V$. Then $A$ acts on $S$ with compact orbits. This can be proved as follows. After complexifying the representation, we may diagonalize the action of the image $\phi(A)$ since it is abelian and consists of semisimple elements. Now, $A$ must be isomorphic to a product of copies of $S^{1}, \mathbb{R}^{\times}$, and $\mathbb{C}^{\times}$. 
Using that every one-dimensional character of these groups has either compact or unbounded image in $\mathbb{C}$, we deduce that every orbit of $A$ on $V \otimes \mathbb{C}$ is either compact or unbounded. In particular, $A$ must act on a compact $S \subset V$ with compact orbits.

Second, if $A$ is an abelian, real algebraic group and $A_{1}, A_{2}$ are cocompact, algebraic, closed subgroups, then $A_{1} \cap A_{2}$ is cocompact in $A$. This is because the fibers of the map

$$
A /\left(A_{1} \cap A_{2}\right) \rightarrow A / A_{1}
$$

are homeomorphic to $A_{1} /\left(A_{1} \cap A_{2}\right) \cong A_{1} A_{2} / A_{2}$, which is compact because it is a closed subset of $A / A_{2}$. More generally, if $A_{1}, \ldots, A_{n}$ is a finite collection of cocompact, algebraic, closed subgroups of a real, abelian algebraic group $A$, then

$$
A / \bigcap_{i=1}^{n} A_{i}
$$

is compact.

Now, back to the proof that $Z(L) / Z(G)$ is compact. By the first remark, $Z(L)$ acts on $\mathcal{O}_{\xi} \cap S_{X}$ with compact orbits for every $\xi \in U$. In particular, $Z(L) /(Z(L) \cap$ $\left.Z_{L}(\xi)\right)$ is compact for all $\xi \in U$. In the above lemma, we showed

$$
\bigcap_{\xi \in U}\left(Z(\mathfrak{l}) \cap Z_{\mathfrak{l}}(\xi)\right)=Z(\mathfrak{g}) .
$$

However, one can clearly choose $\xi_{1}, \ldots, \xi_{k} \in U$ such that the identity still holds when taking the intersection over this finite set. Then, by the second remark,

$$
Z(L) / \bigcap_{i=1}^{k}\left(Z(L) \cap Z_{L}\left(\xi_{i}\right)\right)
$$

is compact. Since $\bigcap_{i=1}^{k}\left(Z(L) \cap Z_{L}\left(\xi_{i}\right)\right)$ is a real algebraic group, it has a finite number of connected components and

$$
Z(L) /\left(\bigcap_{i=1}^{k}\left(Z(L) \cap Z_{L}\left(\xi_{i}\right)\right)\right)_{0}
$$

is also compact where $\left(\bigcap_{i=1}^{k}\left(Z(L) \cap Z_{L}\left(\xi_{i}\right)\right)\right)_{0}$ denotes the identity component. But, since $\bigcap_{i=1}^{k}\left(Z(L) \cap Z_{L}\left(\xi_{i}\right)\right)$ and $Z(G)$ share a Lie algebra, $Z(L) / Z(G)$ is a quotient of

$$
Z(L) /\left(\bigcap_{i=1}^{k}\left(Z(L) \cap Z_{L}\left(\xi_{i}\right)\right)\right)_{0} .
$$

Thus, $Z(L) / Z(G)$ is compact. This completes the proof of Theorem 1.1.

\section{Two EXAmples}

In this section, we give two examples. The author learned these examples from David Vogan.

Example 5.1. Nilpotent orbits in $\mathrm{U}(2,2)$ can be parametrized by signed Young diagrams of signature $(2,2)$ (see page 140 of [3]). A nilpotent orbit of $\mathrm{U}(2,2)$ is noticed iff it corresponds to a signed Young diagram for which any two rows of equal length begin with the same sign. One can read this off of an explicit description of the Levi factor of the centralizer of a nilpotent element in the corresponding 
nilpotent orbit, which in turn can be easily computed from Remark 5.1.18 on page 74 of $[3$ and the discussion on pages $139-140$ of 3 .

On the other hand, which nilpotent orbits of $\mathrm{U}(2,2)$ occur in the wave front cycle of a discrete series can be read off from Example 2.6.7 on page 363 of [14. In Yamamoto's table, the far left column is filled in by sequences of numbers and signs. The entry corresponds to a discrete series representation if the sequence contains only signs. One then observes from Yamamoto's table that the nilpotent orbits corresponding to discrete series for $\mathrm{U}(2,2)$ are precisely the noticed nilpotent orbits of $\mathrm{U}(2,2)$.

Of course, Yamamoto is really computing the associated variety of a discrete series representation. That the associated variety of the representation corresponds to the wave front cycle of the representation under the Kostant-Sekiguchi correspondence is a deep result of $[13$.

Example 5.2. Nilpotent orbits in $\operatorname{Sp}(4, \mathbb{C})$ can be parametrized by partitions of four whose odd parts occur with even multiplicity (see page 70 of [3]). Of these orbits, the principal orbit (corresponding to the trivial partition 4) and the subregular orbit (corresponding to the partition $2^{2}$ ) are the noticed ones as can be read off of page 88 of [3].

However, the wave front cycle of any tempered representations of $\operatorname{Sp}(4, \mathbb{C})$ contains only the principal orbit. Therefore, in this case, the set of nilpotent orbits corresponding to tempered representations is a proper subset of the set of noticed nilpotent orbits.

\section{ACKNOWLEDGMEnTs}

The author would like to thank his advisor, David Vogan, for suggesting the relationship between tempered representations and nilpotent orbits that is explored in this paper. The author would also like to thank David Vogan for many helpful comments and corrections.

\section{REFERENCES}

1. P. Bala, R. Carter, Classes of unipotent elements in simple algebraic groups. I, Math Proc. Cambridge. Philos. Soc., 79 (1976), 401-425. MR0417306 (54:5363a)

2. D. Barbasch, D. Vogan, The local structure of characters, J. Funct. Anal. 37 (1980), 27-55. MR576644 (82e:22024)

3. D. Collingwood, W. McGovern, Nilpotent Orbits in Semisimple Lie Algebras, Van Nostrand Reinhold, (1993), New York, NY. MR1251060 (94j:17001)

4. Harish-Chandra, Some results on an invariant integral on a semi-simple Lie algebra, Ann. of Math. (2), 80 (1964), 551-593. MR0180629(31:4862b)

5. Kirillov, Lectures on the Orbit Method, Graduate Studies in Mathematics, Volume 64, American Mathematical Society, (2004), Providence, RI. MR2069175 (2005c:22001)

6. C. Moeglin, Front d'Onde des representations des groupes classiques p-adiques, American Journal of Mathematics, 118, no. 6 (1996), 1313-1346. MR1420926 (98d:22015)

7. C. Moeglin, J. L. Waldspurger, Modeles de Whittaker degeneres pour des groupes p-adiques, Math Z. 196 (1987), 427-452. MR913667(89f:22024)

8. A. Noël, Nilpotent orbits and theta stable parabolic subalgebras, Journal of Representation Theory, No. 2, (1998), 1-32. MR.1600330 (99g:17023)

9. W. Rossmann, Limit orbits in reductive Lie algebras, Duke Math. J. 49 No. 1 (1982), 215-229. MR650378 (84e:22021)

10. W. Rossmann, Limit characters of reductive Lie groups, Invent. Math. 61 (1980), no. 1, 53-66. MR:587333(81m:22021) 
11. W. Rossmann, Tempered representations and orbits, Duke Math. J. 49 No. 1 (1982), 231-247. MR650379 (84m:22024)

12. W. Rossmann, Picard-Lefschetz theory and characters of a semisimple Lie group, Invent. Math. 121 (1995), 579-611. MR1353309 (96j:22017)

13. W. Schmid, K. Vilonen, Characteristic cycles and wave front cycles of representations of reductive Lie groups, Ann. of Math. (2), 151 (2001), 1071-1118. MR.1779564 (2001j:22017)

14. A. Yamamoto, Orbits in the flag variety and images of the moment map for classical groups I, Journal of Representation Theory, No. 1, (1997), 329-404. MR1479152 (98j:22024)

Department of Mathematics, Massachusetts Institute of Technology, Cambridge, MASSACHusetTs 02139

E-mail address: blharris@math.mit.edu 